\title{
Finite time dividend-ruin models
}

\author{
Kwai Sun Leung, Yue Kuen Kwok* and Seng Yuen Leung ${ }^{\dagger}$ \\ Department of Mathematics, Hong Kong University of Science and Technol- \\ ogy, Clear Water Bay, Hong Kong, China
}

\begin{abstract}
We consider the finite time horizon dividend-ruin model where the firm pays out dividends to its shareholders according to a dividend-barrier strategy and becomes ruined when the firm asset value falls below the default threshold. The asset value process is modeled as a restricted Geometric Brownian process with an upper reflecting (dividend) barrier and a lower absorbing (ruin) barrier. Analytic solutions to the value function of the restricted asset value process are provided. We also solve for the survival probability and the expected present value of future dividend payouts over a given time horizon. The sensitivities of the firm asset value and dividend payouts to the dividend barrier, volatility of the firm asset value and firm's credit quality are also examined.
\end{abstract}

Key words: dividend-ruin model, dividend payouts, reflecting and absorbing barriers, survival probability

JEL Classification: G12, G22

Mathematics Subject Classification (1991): 49K05, 60J70, 91 B30

\section{Introduction}

In this paper, we consider the classical problem of dividend payouts from a firm according to a dividend-barrier strategy, where the excess of the firm asset value above a threshold barrier will be automatically paid out to the shareholders. The underlying stochastic state variable in our dividend-ruin model is the firm asset value, which is modeled as a restricted Geometric

\footnotetext{
*Correspondence author: e-mail: maykwok@ust.hk.

${ }^{\dagger}$ Present address: Morgan Stanley Dean Witter Asia Ltd.
} 
Brownian motion with a lower absorbing barrier and an upper reflecting barrier. The absorbing state represents the default of the firm when the asset value reaches the default threshold. The upper reflecting barrier models the dividend-barrier policy. We present the partial differential equation formulation of the restricted asset value process, in particular, the prescription of the auxiliary condition associated with the dividend barrier. Under the assumption of constant interest rate, we solve for the value function of the asset value process, survival probability and expected present value of future dividend payouts from the risky firm over a finite time horizon. We also examine the sensitivities of the firm asset value and dividend payouts to the dividend barrier, volatility of the firm asset value and firm's credit quality.

In the actuarial science literature, the dividend-ruin problem can be considered as a special case of the general consumption-investment problem. There have been numerous papers on various forms of the perpetual dividendruin models. A recent survey of the dividend control models is given by Taksar (2000). In a typical model, the surplus process is modeled by a compound Poisson process or a Brownian process with drift (Paulsen and Gjessing, 1997). The dividend policy can be a constant payout at a dynamic rate that may be dependent on the current surplus (Asmussen and Taksar, 1997). Shreve et al. (1984) show that under some general assumptions the optimal dividend policy would be the barrier strategy, that is, the firm pays out the excess surplus when the asset value goes beyond a dividend-barrier $B$. In some recent papers on dividend-ruin models, the authors consider the optimal dividend distribution subject to constraints on risk controls. Paulsen (2003) includes the solvency requirement on the allowable dividend policy. In his model, the firm is not allowed to pay dividend when the survival probability over a given time period falls below a pre-set non-tolerant level. Choulli et al. (2003) add a control in their perpetual dividend-ruin model to monitor the firm's risk (for example, through reinsurance). The control can decrease simultaneously the drift and diffusion coefficients in the underlying surplus process.

Our dividend-ruin model follows quite closely the formulation proposed by Gerber and Shiu (2003), where the firm asset value is used as the underlying process. The firm asset value approach is a slight departure from most dividend-ruin models in the literature, where the surplus process has been commonly used as the underlying process. These surplus process models assume bankruptcy to occur when the surplus hits the zero value. We prefer the use of the asset value process in our model since the asset value 
process is more directly related to the capital structure of the firm and the firm's stock price dynamics. Our model assumes that there exists an exogenously imposed default threshold such that the firm defaults (is ruined) when the asset value falls below this threshold. The default threshold can be deduced from the liabilities of the firm (obtainable from the balance sheet information). Our model framework is related to the structural models that analyze defaultable bonds (Longstaff and Schwartz, 1985). The industrial KMV software code puts the structural models into practice in analyzing the creditworthiness of a risky firm (Crosbe and Bohn, 1993).

In our model, we assume a dividend barrier strategy where the firm pays out the excess of the asset value above the constant dividend barrier as dividends. The dividend barrier may be determined by the combination of optimality in dividend distribution and solvency requirement as in Paulsen (2003). Assuming that the firm follows the dividend barrier policy, the dividend barrier becomes a reflecting barrier for the asset value process. Together with the absorbing barrier at the default threshold, the asset value process becomes a restricted process with an upper reflecting (dividend) barrier and a lower absorbing (ruin) barrier. While all earlier dividend-ruin models compute the expected present value of future dividends over perpetuity, we derive closed form formulas that give the survival probability and the expected present value of future dividends over a finite time period.

This paper is structured as follows. In the next section, we present the formulation of our dividend-ruin model with the firm asset value restricted by a lower ruin barrier and an upper dividend barrier. The restricted asset value process is seen to include both the lookback and barrier features. In Section 3 , we present the partial differential equation formulation for the value function of the firm value process, and provide the eigenfunction solution of the governing differential equation. We also show how to obtain a fairly accurate analytic approximation price formula. In Section 4, we present the solution of the survival probability and the expected present value of future dividends over a finite time horizon. We also examine the dependence of the expected amount of dividend payouts and survival probability on the dividend barrier, ruin barrier and firm's creditworthiness. Concluding remarks and summaries are presented in the last section. 


\section{Formulation of the dividend-ruin model}

In this paper, we use the firm asset value process rather than the surplus process as the underlying process to model the wealth dynamics of the firm. As usual, we start with a filtered probability space $\left(\Omega, \mathcal{F}, \mathcal{F}_{t}, \mathbb{P}\right)$ and a standard Brownian motion $Z_{t}$ adapted to the filtration $\mathcal{F}_{t}$. Here, $\mathbb{P}$ is the probability measure. Let $A_{t}$ denote the asset value of a firm which follows the Geometric Brownian motion, where

$$
\frac{\mathrm{d} A_{t}}{A_{t}}=\mu \mathrm{d} t+\sigma \mathrm{d} Z_{t} .
$$

Here, $\mu$ is the constant drift rate, $\sigma^{2}$ is the variance rate and $Z_{t}$ is the standard Brownian motion. We write $A_{t}=A_{0} e^{W_{t}}$, where $A_{0}$ is the asset value at some reference "zeroth" time. Here, $W_{t}$ is a Brownian motion with drift rate $\alpha=\mu-\frac{\sigma^{2}}{2}$ and variance rate $\sigma^{2}$ defined by

$$
W_{t}=\alpha t+\sigma Z_{t}
$$

We use $\underline{\mathrm{W}}_{t_{1}}^{t_{2}}$ and $\bar{W}_{t_{1}}^{t_{2}}$ to denote the respective minimum value and maximum value of the Brownian process $W_{t}$ over the time period $\left[t_{1}, t_{2}\right]$. Suppose we write $\underline{A}_{0}^{T}$ and $\bar{A}_{0}^{T}$ to denote the minimum value and maximum value of the asset value process over the time period $[0, T]$, and let $t$ denote the current time where $t \in[0, T]$, we then have

$$
\begin{aligned}
& \underline{A}_{0}^{T}=A_{0} e^{\min _{0 \leq s \leq T} W_{s}}=\min \left(A_{0} e^{\frac{W_{0}^{t}}{}}, A_{t} e^{\underline{W}_{t}^{T}}\right) \\
& \bar{A}_{0}^{T}=A_{0} e^{\max _{0 \leq s \leq T} W_{s}}=\max \left(A_{0} e^{\bar{W}_{0}^{t}}, A_{t} e^{\bar{W}_{t}^{T}}\right) .
\end{aligned}
$$

Here, $\underline{W}_{0}^{t}$ and $\bar{W}_{0}^{t}$ are the realized extremum values over $[0, t]$ that are already known at the current time $t$ while $\underline{W}_{t}^{T}$ and $\bar{W}_{t}^{T}$ are the stochastic lookback state variables.

The formulation of our proposed dividend-ruin model follows closely to that of the modified asset value process presented by Gerber and Shiu (2003). Let $L$ denote the liability or default threshold such that the firm becomes default when the asset value $A_{t}$ falls to $L$. The liability level $L$ may be visualized as the lower absorbing barrier or the knock-out barrier of the asset value process. On the other hand, the firm pays out dividends to shareholders according to a dividend barrier strategy with an upper barrier 
$B$. Whenever the asset value rises to $B$, the excess amount will be paid out as dividends. Under such dividend strategy, the restricted asset value can never go above $B$. Hence, the barrier level $B$ may be considered as an upper reflecting barrier.

Subject to the possibilities of ruin and dividend payouts, the asset value process becomes restricted with a lower absorbing barrier and an upper reflecting barrier. Let $\widetilde{A}_{t}$ denote the corresponding modified (or restricted) asset value process. One may visualize the dividend payouts as withdrawal of portion of the firm's asset so that the remaining firm's asset value always stays at or below $B$ (Gerber and Shiu, 2003). Over the finite period $[0, t]$, the fraction of the firm's asset remaining is given by $\min \left(1, \frac{B}{\bar{A}_{0}^{t}}\right)$. We define the non-ruined modified asset value $\widehat{A}_{t}$ at time $t$ to be

$$
\widehat{A_{t}}=A_{t} \min \left(1, \frac{B}{\bar{A}_{0}^{t}}\right)
$$

and denote the running minimum value of $\widehat{A}_{t}$ over the time interval $\left[t_{1}, t_{2}\right]$ by

$$
\widehat{\widehat{A}}_{t_{1}}^{t_{2}}=\min _{t_{1} \leq t \leq t_{2}} \widehat{A}_{t}
$$

Hence, the modified (or restricted) asset value at time $T$ is given by

$$
\widetilde{A}_{T}=\widehat{A}_{T} \mathbf{1}_{\left\{\underline{\hat{A}}_{0}^{T}>L\right\}}
$$

The indicator function $\mathbf{1}_{\left\{\underline{\underline{A}}_{0}^{T}>L\right\}}$ is included in $\widetilde{A}_{T}$ to reflect the ruin feature that the asset value becomes zero when the modified asset value $\widehat{A}_{t}$ at any intermediate time $t$ falls to $L$.

\section{Value function of firm value process}

Our dividend-ruin model is defined over the finite time horizon $[0, T]$. Let $t$ denote the current time, where $t \in[0, T]$. We are interested to compute the expected present value of the modified asset value at the future time $T$. Let $A$ denote the asset value $A_{t}$ at time $t$ and $V_{i n}\left(A, \tau ; \underline{A}_{0}^{t}, \bar{A}_{0}^{t}, L, B\right)$ denote the 
corresponding in-progress value function, with dependence on $A, \tau=T-t$ and parameter values $\underline{A}_{0}^{t}, \bar{A}_{0}^{t}, L$ and $B$. By definition, $V_{\text {in }}$ is given by

$$
V_{i n}\left(A, \tau ; \underline{A}_{0}^{t}, \bar{A}_{0}^{t}, L, B\right)=\mathbb{E}_{t}\left[e^{-r \tau} \widetilde{A}_{T}\right]
$$

where $\mathbb{E}_{t}$ denotes the expectation under the probability measure $\mathbb{P}$ conditional on the filtration $\mathcal{F}_{t}$. The above expectation representation is complicated by the presence of the realized minimum and maximum value of the firm value process over $[0, t]$. Provided that $\underline{\widehat{A}}_{0}^{t}>L$ and $\bar{A}_{0}^{t} \leq B, \widehat{A}_{t}$ is the same as $A_{t}$. We define $V(A, \tau ; L, B)$ as the "initiation-state" value function with no dependence on $\underline{A}_{0}^{t}$ and $\bar{A}_{0}^{t}$, corresponding to the state where $A_{t}$ has not reached either the lower absorbing barrier or the upper reflecting barrier within $[0, t]$. The following relation between $V_{i n}$ and $V$ can be deduced [similar results can be found in Chu and Kwok (2004)]:

$$
V_{i n}\left(A, \tau ; \underline{A}_{0}^{t}, \bar{A}_{0}^{t}, L, B\right)= \begin{cases}V\left(\frac{B}{\bar{A}_{0}^{t}} A, \tau ; L, B\right) & \text { if } \widehat{\widehat{A}}_{0}^{t}>L \text { and } \bar{A}_{0}^{t}>B \\ V(A, \tau ; L, B) & \text { if } \widehat{\widehat{A}}_{0}^{t}>L \text { and } \bar{A}_{0}^{t} \leq B \\ 0 & \text { if } \underline{\widehat{A}}_{0}^{t} \leq L\end{cases}
$$

The mathematical proof of the first relation in Eq. (3.2) is presented in Appendix A, while other relations can be derived in a similar manner.

The above relations agree with the following financial intuition. When $\underline{\widehat{A}}_{0}^{t}>L$ and $\bar{A}_{0}^{t}>B$, the firm remains alive and dividends have been paid out to the shareholders. The fraction of the original asset value remaining is $B / \bar{A}_{0}^{t}$ so that the modified firm asset value process becomes $\left(B / \bar{A}_{0}^{t}\right) A_{t}$. Referring to the non-ruined modified asset value $\widehat{A}_{t}$, the dividend barrier remains to be $B$ and ruined barrier remains to be $L$, thus we establish the first relation in Eq. (3.2). When $\underline{\widehat{A}}_{0}^{t}>L$ and $\bar{A}_{0}^{t} \leq B$, the firm remains alive and no dividends have been paid out. In this case, there is no modification to the firm asset value. Lastly, when $\underline{A}_{0}^{t} \leq L$, the firm has ruined already so that $V_{\text {in }}=0$.

Next, we present the differential equation formulation of the "initiationstate" value function $V(A, \tau ; L, B)$. Shreve et al. (1984) show that the dividend payout can be considered as a non-decreasing "withdrawal" process. The firm asset process is controlled by subtracting off the dividend payoff and the controlled process is absorbed when it reaches the default barrier. Shreve 
et al. (1984) prove that under the perpetuality assumption the controlled process is reflected at the dividend barrier and absorbed at the default barrier. In their differential equation formulation, the absorbing barrier gives the Dirichlet condition while the reflecting barrier is prescribed by the Neumann condition. Extending to the finite-time horizon model, the partial differential equation formulation for $V(A, \tau ; L, B)$ is given by

$$
\frac{\partial V}{\partial \tau}=\mathcal{L} V, \quad L<A<B, \tau>0
$$

where

$$
\mathcal{L}=\frac{\sigma^{2}}{2} A^{2} \frac{\partial^{2}}{\partial A^{2}}+\mu A \frac{\partial}{\partial A}-r
$$

The auxiliary conditions are:

$$
\begin{aligned}
& V(L, \tau)=0 \text { and } \frac{\partial V}{\partial A}(B, \tau)=0 \quad \text { for all } \tau, \\
& V(A, 0)=A, \quad L<A<B .
\end{aligned}
$$

To justify the validity of the above auxiliary conditions in our finite time horizon model, one may follow a similar analytic technique used in the derivation of the differential equation formulation of the expected present value of dividends (see Section 4).

\section{Analytic solution}

Given the above partial differential equation formulation of $V(A, \tau)$, we would like to derive its analytical solution in the form of an infinite series. We define

$$
x=\ln \frac{A}{B} \quad \text { and } \quad \ell=\ln \frac{L}{B},
$$

it can be shown that $V(A, \tau)$ can be expressed in the form

$$
V(A, \tau)=e^{-r \tau} \int_{\ell}^{0} B e^{y} G(x, \tau ; y) \mathrm{d} y, \quad A=B e^{x} .
$$

The Green function $G(x, \tau ; y)$ is governed by

$$
\frac{\partial G}{\partial \tau}=\frac{\sigma^{2}}{2} \frac{\partial^{2} G}{\partial x^{2}}+\alpha \frac{\partial G}{\partial x}, \quad \ell<x<0 \quad \text { and } \quad \tau>0
$$


with auxiliary conditions:

$$
\begin{gathered}
G(\ell, \tau ; y)=0 \quad \text { and } \quad \frac{\partial G}{\partial x}(0, \tau ; y)=0 \\
G(x, 0 ; y)=\delta(x-y) .
\end{gathered}
$$

The analytic representation of the Green function with mixed DirichletNeumann boundary conditions is less well known compared to that of the counterpart with Dirichlet condition at both boundaries. Recall that the Green function with double Dirichlet conditions can be represented by an infinite series involving either the normal kernel functions or the eigenfunctions (whose analytic form is a product of exponential function in time and sinusoidal function in space). In a similar manner, two different series representations of $G$ can be found. The solution of $G$ in a series expansion of eigenfunctions has been derived by Domine (1996). In this paper, we derive another analytic representation of the solution in terms of the parabolic cylinder functions, the details of which are relegated to Appendix B.

Suppose we adopt the eigenfunction solution of the Green function, the evaluation of the integral in Eq. (3.6) gives the following solution to $V(A, \tau)$ :

$$
\begin{aligned}
V(A, \tau)= & B e^{-r \tau}\left(\frac{B}{A}\right)^{\alpha / \sigma^{2}}\left[d_{0} e^{-\alpha^{2} \tau / 2 \sigma^{2}}-\sum_{n=1}^{\infty} \frac{d_{n}}{\frac{\ell}{2}+\frac{\sigma^{2}}{2 \alpha} \cos ^{2} \lambda_{n}}\right. \\
& \left.\exp \left(-\left(\frac{\alpha^{2}}{2 \sigma^{2}}+\frac{\sigma^{2} \lambda_{n}^{2}}{2 \ell^{2}}\right) \tau\right) \sin \left(\frac{\lambda_{n}}{\ell}\left(\ell-\ln \frac{A}{B}\right)\right)\right],
\end{aligned}
$$

where

$$
d_{n}=\frac{1+\frac{\alpha}{\sigma^{2}}}{\left(1+\frac{\alpha}{\sigma^{2}}\right)^{2}+\frac{\lambda_{n}^{2}}{\ell^{2}}}\left\{\sin \lambda_{n}-\frac{\lambda_{n}}{\left(1+\frac{\alpha}{\sigma^{2}}\right) \ell}\left[e^{\left(1+\frac{\alpha}{\sigma^{2}}\right) \ell}-\cos \lambda_{n}\right]\right\} .
$$

Here, $\lambda_{n}$ is the solution to

$$
\tan \lambda=-\frac{\sigma^{2} \lambda}{\alpha \ell}
$$

for $\lambda_{n} \in\left(n \pi,\left(n+\frac{1}{2}\right) \pi\right), n=0,1,2, \cdots$.

Note that $d_{0}$ takes different functional forms depending on the sign of $\alpha+\frac{\sigma^{2}}{\ell}$. We have 
(i) $\alpha+\frac{\sigma^{2}}{\ell}<0$

$$
\begin{aligned}
d_{0}= & -\frac{\exp \left(-\frac{\lambda_{0}^{2} \sigma^{2} \tau}{2 \ell^{2}}\right) \sin \frac{\lambda_{0}(\ell-x)}{\ell}}{\frac{\ell}{2}+\frac{\sigma^{2}}{2 \alpha} \cos ^{2} \lambda_{0}} \frac{1+\frac{\alpha}{\sigma^{2}}}{\left(1+\frac{\alpha}{\sigma^{2}}\right)^{2}+\frac{\lambda_{0}^{2}}{\ell^{2}}} \\
& \left\{\sin \lambda_{0}-\frac{\lambda_{0}}{\ell\left(1+\frac{\alpha}{\sigma^{2}}\right)}\left[e^{\left(1+\frac{\alpha}{\sigma^{2}}\right) \ell}-\cos \lambda_{0}\right]\right\} ;
\end{aligned}
$$

(ii) $\alpha+\frac{\sigma^{2}}{\ell}=0$

$$
d_{0}=\frac{3(\ell-x)}{\ell^{3}\left(1+\frac{\alpha}{\sigma^{2}}\right)}\left\{\frac{\left[e^{\left(1+\frac{\alpha}{\sigma^{2}}\right) \ell}-1\right]}{1+\frac{\alpha}{\sigma^{2}}}-\ell\right\}
$$

(iii) $\alpha+\frac{\sigma^{2}}{\ell}>0$

$$
\begin{aligned}
d_{0}= & \frac{\exp \left(\frac{\eta^{2} \sigma^{2} \tau}{2 \ell^{2}}\right)}{\frac{\sigma^{2}}{2 \alpha} \cosh ^{2} \eta-\frac{\ell}{2}} \sinh \frac{\eta(\ell-x)}{\ell} \frac{1+\frac{\alpha}{\sigma^{2}}}{\left(1+\frac{\alpha}{\sigma^{2}}\right)^{2}-\frac{\eta^{2}}{\ell^{2}}} \\
& \left\{\sinh \eta+\frac{\eta}{\ell\left(1+\frac{\alpha}{\sigma^{2}}\right)}\left[\cosh \eta-e^{\left(1+\frac{\alpha}{\sigma^{2}}\right) \ell}\right]\right\},
\end{aligned}
$$

where $\eta$ is the solution to $\tanh \eta=-\frac{\sigma^{2} \eta}{\alpha \ell}$.

\section{Analytic approximation formula}

It is well known that the rate of convergence of the eigenfunction series to the exact solution is relatively slow. Also, the accurate determination of the eigenvalues $\lambda_{n}$ poses difficulties in the numerical evaluation procedure. It is desirable to express the solution in the form of the exponential kernel functions, like that of the density function of the Brownian process with twosided absorbing barriers. While it is not possible to express the asset value function $V$ in terms of the exponential kernel functions, we manage to obtain an analytic approximation to $V$ whose analytic representation involves the 
exponential kernel functions only. Let $\tau_{B}=\inf \left\{t \geq 0, A_{t}=B\right\}$, and $\mathrm{E}$ denote the expectation under the measure $\mathbb{P}$, and recall [see Eq. 2.4a)]

$$
\widehat{A}_{T}=A_{T} \min \left(1, \frac{B}{\bar{A}_{0}^{T}}\right) \quad \text { and } \quad \widehat{A}_{t}=A_{t} \min \left(1, \frac{B}{\bar{A}_{0}^{t}}\right)
$$

the "initiation-state" asset value function for a term $T$ can be expressed as

$$
\begin{aligned}
& V(A, T ; L, B) \\
& =e^{-r T} \mathbb{E}\left[\widehat{A}_{T} \mathbf{1}_{\left\{\underline{\widehat{A}}_{0}^{T}>L\right\}}\right] \\
& =e^{-r T} \mathbb{E}\left[A_{T} \mathbf{1}_{\left\{\underline{A}_{0}^{T}>L\right\}} \mathbf{1}_{\left\{\bar{A}_{0}^{T} \leq B\right\}}\right] \\
& +e^{-r T} \mathbb{E}\left[A_{T} \frac{B}{\bar{A}_{0}^{T}} \mathbf{1}_{\left\{B<\bar{A}_{0}^{T}\right\}} \mathbf{1}_{\left\{\underline{\hat{A}}_{0}^{T}>L\right\}}\right] \\
& =e^{-r T} \mathbb{E}\left[A_{T} \mathbf{1}_{\left\{\underline{A}_{0}^{T}>L\right\}} \mathbf{1}_{\left\{\bar{A}_{0}^{T} \leq B\right\}}\right] \\
& +e^{-r T} \mathbb{E}\left[A_{T} \frac{B}{\bar{A}_{0}^{T}} \mathbf{1}_{\left\{B<\bar{A}_{0}^{T}\right\}} \mathbf{1}_{\left\{\min \left(\underline{A}_{0}^{\tau_{B}}, \min _{\tau_{B} \leq t \leq T} A_{t} \frac{B}{\bar{A}_{0}^{t}}\right)>L\right\}}\right] \\
& =e^{-r T} \mathbb{E}\left[\widehat{A}_{T} \mathbf{1}_{\left\{\underline{A}_{0}^{T}>L\right\}}\right] \\
& +\mathbb{E}\left[A_{T} \frac{B}{\bar{A}_{0}^{T}} \mathbf{1}_{\left\{B<\bar{A}_{0}^{T}\right\}} \mathbf{1}_{\left\{\underline{A}_{0}^{\tau_{B}}>\min _{\tau_{B} \leq t \leq T} A_{t} \frac{B}{\bar{A}_{0}^{t}}\right\}}\right. \\
& \left.\left(\mathbf{1}_{\left\{\min _{0 \leq t \leq T} A_{t} \frac{B}{\bar{A}_{0}^{t}}>L\right\}}-\mathbf{1}_{\left\{\underline{A}_{0}^{T}>L\right\}}\right)\right] \text {. }
\end{aligned}
$$

The second term in the last expression is small when the expectation of the difference between the two indicator functions $\mathbb{E}\left[\mathbf{1}_{\left\{\min _{0 \leq t \leq T} A_{t} \frac{B}{\bar{A}_{0}^{t}}>L\right\}}-\mathbf{1}_{\left\{\underline{A}_{0}^{T}>L\right\}}\right]$ is small. This is true when $A$ is sufficiently close to $L$.

We set $V_{a}(A, T ; L, B)$ to be $e^{-r T} \mathbb{E}\left[\widehat{A}_{T} \mathbf{1}_{\left\{\underline{A}_{0}^{T}>L\right\}}\right]$, which is taken to be an analytic approximation to $V(A, T ; L, B)$. Since the original indicator function $\mathbf{1}_{\left\{\underline{\hat{A}}_{0}^{T}>L\right\}}$ in $V$ has been replaced by $\mathbf{1}_{\left\{\underline{A}_{0}^{T}>L\right\}}$ in $V_{a}$, it becomes possible to express $V_{a}$ in terms of the density function of the restricted Brownian 
process with two-sided absorbing barriers. After performing several derivation steps in double integration (see Appendix $\mathrm{C}$ for details), we obtain the following representation for $V_{a}$ :

$$
V_{a}(A, T ; L, B)=e^{-r T} B \int_{K}^{\infty} \int_{0}^{M} e^{x} e^{-M} f\left(x, M, T ; x_{0}\right) \mathrm{d} x \mathrm{~d} M,
$$

where $K=\ln \frac{B}{L}$ and $f\left(x, M, T ; x_{0}\right)$ is the density function of the terminal value $W_{T}$ of the Brownian motion with drift $\alpha$ that is subject to two-sided absorbing barriers. It is known that

$$
\begin{gathered}
f\left(x, M, T ; x_{0}\right)=\mathbb{P}\left[W_{T} \in \mathrm{d} x, \bar{W}_{0}^{T}<M, \underline{\left.W_{0}^{T}>0 \mid W_{0}=x_{0}\right]}\right. \\
=\exp \left(\frac{2 \alpha\left(x-x_{0}\right)-\alpha^{2} T}{2 \sigma^{2}}\right) \\
\sum_{n=-\infty}^{\infty} \frac{1}{\sqrt{2 \pi \sigma^{2} T}}\left[\exp \left(-\frac{\left(x-x_{0}-2 n M\right)^{2}}{2 \sigma^{2} T}\right)\right. \\
\left.-\exp \left(-\frac{\left(x+x_{0}-2 n M\right)^{2}}{2 \sigma^{2} T}\right)\right] .
\end{gathered}
$$

By evaluating the double integral in Eq. (3.12), we obtain

$$
V_{a}(A, T ; L, B)=e^{-r T} B e^{-\frac{1}{2 \sigma^{2}}\left(\alpha^{2} T+2 \alpha x_{0}\right)} \sum_{n=-\infty}^{\infty}\left[I_{1}(n)+I_{2}(n)\right],
$$

where

(i) $n \neq 0$

$$
\begin{aligned}
I_{1}(n)= & \Psi_{n}\left(-\frac{n}{|n|},-x_{0}-\frac{\sigma^{2} T}{2|n|}-2|n| K, 1+\frac{\alpha}{\sigma^{2}}-\frac{1}{2 n}, 0, K,-x_{0}\right) \\
& -\Psi_{n}\left(-\frac{n}{|n|}, x_{0}-\frac{\sigma^{2} T}{2|n|}-2|n| K, 1+\frac{\alpha}{\sigma^{2}}-\frac{1}{2 n}, 0, K, x_{0}\right), \\
I_{2}(n)= & \Psi_{n}\left((2 n-1) \frac{n}{|n|},-x_{0}-\frac{\sigma^{2} T}{2|n|}, 1+\frac{\alpha}{\sigma^{2}}-\frac{1}{2 n}, K, \infty, \frac{n}{|n|} x_{0}\right) \\
& -\Psi_{n}\left((2 n-1) \frac{n}{|n|}, x_{0}-\frac{\sigma^{2} T}{2|n|}, 1+\frac{\alpha}{\sigma^{2}}-\frac{1}{2 n}, K, \infty, \frac{n}{|n|} x_{0}\right),
\end{aligned}
$$




$$
\begin{aligned}
\Psi_{n}(a, b, c, d, f, g)= & \frac{1}{2 n} \frac{e^{\frac{g}{2 n}+\frac{1}{2}\left(\frac{1}{2 n}\right)^{2} \sigma^{2} T}}{c}\left\{e^{c f} N\left(\frac{b-a f}{\sigma \sqrt{T}}\right)-e^{c d} N\left(\frac{b-a d}{\sigma \sqrt{T}}\right)\right. \\
& +e^{\frac{b c}{a}+\frac{1}{2}\left(\frac{c}{a}\right)^{2} \sigma^{2} T}\left[N\left(\frac{a f-\left(b+\frac{c}{a} \sigma^{2} T\right)}{\sigma \sqrt{T}}\right)\right. \\
& \left.\left.-N\left(\frac{a d-\left(b+\frac{c}{a} \sigma^{2} T\right)}{\sigma \sqrt{T}}\right)\right]\right\}
\end{aligned}
$$

(ii) $n=0$

$$
\begin{gathered}
I_{1}(0)=\frac{L}{B}\left[\Phi\left(1, x_{0}, 1+\frac{\alpha}{\sigma^{2}}, 0, K\right)-\Phi\left(1,-x_{0}, 1+\frac{\alpha}{\sigma^{2}}, 0, K\right)\right], \\
I_{2}(0)=\Phi\left(1, x_{0}, \frac{\alpha}{\sigma^{2}}, K, \infty\right)-\Phi\left(1,-x_{0}, \frac{\alpha}{\sigma^{2}}, K, \infty\right) \\
\Phi(a, b, c, d, f)=\frac{1}{a} e^{\frac{b c}{a}+\frac{1}{2}\left(\frac{c}{a}\right)^{2} \sigma^{2} T} \\
{\left[N\left(\frac{a f-\left(b+\frac{c}{a} \sigma^{2} T\right)}{\sigma \sqrt{T}}\right)-N\left(\frac{a d-\left(b+\frac{c}{a} \sigma^{2} T\right)}{\sigma \sqrt{T}}\right)\right] .}
\end{gathered}
$$

Here, $N(\cdot)$ denotes the cumulative standard normal distribution function.

We performed numerical calculations to testify the accuracy of the analytic approximation formula. In Figure 1, we show the plot of the asset value function $V(A, T ; L, B)$ against $\ln (A / L)$. The model parameter values used in the calculations are: $r=0.08, \sigma=0.15, T=1, \mu=0.08, L=0.2, B=1.2$. We compare the numerical approximation values of $V$ obtained from the analytic approximation formula (3.14) with the numerical results obtained by solving the partial differential equation for $V$ [see Eqs. $(3.5 \mathrm{a}, \mathrm{b})]$ using the finite difference algorithm. As shown by the two curves in Figure 1, the finite difference solution to the asset value function agrees very well with the solution obtained from the analytic approximation formula even at relatively high value of $A / L$. As revealed from Figure $1, V(A, T ; L, B)$ is an increasing function of $A$, with a higher value of $\frac{\partial V}{\partial A}$ when $A$ is closer to the ruin barrier $L$ and a lower value of $\frac{\partial V}{\partial A}$ when $A$ is closer to the dividend barrier $B$.

We also examine the dependence of $V(A, T ; L, B)$ on the dividend barrier $B$. Figure 2 shows the plot of $V(A, T ; L, B)$ against $B$ with varying values 
of the ruin barrier $L$. Here, we take $A=1$ and use the same set of model parameter values as those in Figure 1 in the numerical calculations. When the ratio $L / A$ is small, corresponding to a high credit quality of the firm, the asset value function $V$ is seen to be quite insensitive to the level of ruin barrier. This is revealed by the overlapping of the asset value curves for $L=0.4$ and $L=0.6$ where $A$ is taken to be 1 . For a fixed value of $L$, the asset value function is an increasing function of dividend barrier since the dividend payout is less with a higher dividend barrier. The curves in Figure 2 illustrate the phenomenon of the high sensitivity of $V$ to the dividend barrier level.

\section{Expected present value of dividends and survival probability}

In this section, we would like to derive the analytic formulation of the expected present value of future dividends and the survival probability, and examine their dependence on the creditworthiness of the firm, dividend barrier and ruin barrier.

Recall that $\widehat{A}_{t}$ as defined in Eq. (2.4a) represents the non-ruined modified asset value at time $t$, which is the asset value process after dividends. Let $\mathrm{d} C_{t}$ denote the non-negative amount of dividends paid in the time interval $[t, t+\mathrm{d} t)$, and $C_{t}$ is adapted to the filtration $\mathcal{F}_{t}$. The stochastic differential equation for $\widehat{A}_{t}$ takes the form

$$
\mathrm{d} \widehat{A}_{t}=\mu \widehat{A}_{t} \mathrm{~d} t+\sigma \widehat{A}_{t} \mathrm{~d} Z_{t}-\mathrm{d} C_{t}
$$

with $\widehat{A}_{0}=A$. Let $\widehat{\tau}$ denote the first passage time of $\widehat{A}_{t}$ to the ruin barrier $L$, that is,

$$
\widehat{\tau}=\inf \left\{t \geq 0, \widehat{A}_{t}=L\right\} .
$$

Let $F(A, T ; L, B)$ denote the expected present value of dividends at time zero over a term $T$ subject to ruin at a lower barrier $L$ and dividend payout at an upper barrier $B$. We then have

$$
F(A, T)=\mathbb{E}\left[\int_{0}^{T \wedge \widehat{\tau}} e^{-r u} \mathrm{~d} C_{u}\right],
$$


where $\mathrm{E}$ denotes the expectation under the measure $\mathbb{P}$. By considering a function $\phi(A, \tau) \in C^{2,1}((L, B) \times(0, \infty))$ that satisfies

$$
\frac{\partial \phi}{\partial \tau}(A, \tau)=\frac{\sigma^{2}}{2} A^{2} \frac{\partial^{2} \phi}{\partial A^{2}}(A, \tau)+\mu A \frac{\partial \phi}{\partial A}(A, \tau)-r \phi(A, \tau)
$$

with auxiliary conditions:

$$
\begin{aligned}
\phi(A, 0) & =0, \quad L<A<B, \\
\phi(L, \tau) & =0 \quad \text { and } \quad \frac{\partial \phi}{\partial A}(B, \tau)=1, \quad \tau>0,
\end{aligned}
$$

we would like to show that

$$
F(A, T)=\phi(A, T) .
$$

To establish the result in Eq. (4.6), we follow the procedure outlined in Freidlin (1985). First, we apply the Ito calculus to obtain

$$
\begin{aligned}
& e^{-r(\tau \wedge \widehat{\tau})} \phi\left(\widehat{A}_{\tau \wedge \widehat{\tau}}, T-\tau \wedge \widehat{\tau}\right) \\
& =\phi(A, T)+\int_{0}^{\tau \wedge \widehat{\tau}} e^{-r u}\left[-\frac{\partial \phi}{\partial \tau}\left(\widehat{A}_{u}, T-u\right)+\frac{\sigma^{2}}{2} A^{2} \frac{\partial^{2} \phi}{\partial A^{2}}\left(\widehat{A}_{u}, T-u\right)\right. \\
& \left.\quad+\mu A \frac{\partial \phi}{\partial A}\left(\widehat{A}_{u}, T-u\right)-r \phi\left(\widehat{A}_{u}, T-u\right)\right] \mathrm{d} u \\
& -\int_{0}^{\tau \wedge \widehat{\tau}} e^{-r u} \frac{\partial \phi}{\partial A}\left(\widehat{A}_{u}, T-u\right) \mathrm{d} C_{u} \\
& +\int_{0}^{\tau \wedge \widehat{\tau}} e^{-r u} \frac{\partial \phi}{\partial A}\left(\widehat{A}_{u}, T-u\right) \widehat{A}_{u} \sigma \mathrm{d} Z_{u} .
\end{aligned}
$$

Next, we set $\tau=T$ and take the expectation under $\mathbb{P}$ on both sides of the above equation. By enforcing the partial differential equation for $\phi$ and the boundary condition: $\frac{\partial \phi}{\partial A}=1$, we obtain

$$
\begin{aligned}
\phi(A, T)= & \mathbb{E}\left[e^{-r(T \wedge \widehat{\tau})} \phi\left(\widehat{A}_{T \wedge \widehat{\tau}}, T-T \wedge \widehat{\tau}\right)\right] \\
& +\mathbb{E}\left[\int_{0}^{T \wedge \widehat{\tau}} e^{-r u} \mathrm{~d} C_{u}\right]
\end{aligned}
$$


The last term is simply $F(A, T)$. It suffices to show that the first term vanishes. The first term can be split into two terms, namely,

$$
\begin{aligned}
& \mathbb{E}\left[e^{-r(T \wedge \widehat{\tau})} \phi\left(\widehat{A}_{T \wedge \widehat{\tau}}, T-T \wedge \widehat{\tau}\right)\right] \\
= & \mathbb{E}\left[e^{-r T} \phi\left(\widehat{A}_{T}, 0\right) \mathbf{1}_{\{\widehat{\tau} \geq T\}}\right]+\mathbb{E}\left[e^{-r \widehat{\tau}} \phi(L, T-\widehat{\tau}) \mathbf{1}_{\{\widehat{\tau}<T\}}\right] .
\end{aligned}
$$

Both terms are seen to be zero by virtue of the auxiliary conditions: $\phi(A, 0)=$ 0 and $\phi(L, \tau)=0, \tau>0$. Hence, we obtain the result in Eq. (4.6).

Next, we would like to establish the relation between $F(A, T)$ and $V(A, T)$. If we let

$$
\psi(A, \tau)=\phi(A, \tau)-A+L,
$$

then the governing equation for $\psi(A, \tau)$ becomes

$$
\frac{\partial \psi}{\partial \tau}=\frac{\sigma^{2}}{2} A^{2} \frac{\partial^{2} \psi}{\partial A^{2}}+\mu A \frac{\partial \psi}{\partial A}-r \psi+(\mu-r) A+r L
$$

with auxiliary conditions:

$$
\begin{aligned}
& \psi(A, 0)=L-A, \quad L<A<B, \\
& \psi(L, \tau)=0 \quad \text { and } \quad \frac{\partial \psi}{\partial A}(B, \tau)=0 .
\end{aligned}
$$

Now, the two boundary conditions $(4.12 \mathrm{a}, \mathrm{b})$ are homogeneous, similar to those of the asset value function $V(A, \tau)$ [see Eq. (3.5b)]. It can be shown that the solution to $\psi(A, \tau)$ admits the following stochastic representation:

$$
\begin{aligned}
\psi(A, \tau)= & \mathbb{E}\left[e^{-r \tau}\left(L-\widehat{A}_{T}\right) \mathbf{1}_{\{\tau<\widehat{\tau}\}}\right] \\
& +\mathbb{E}\left[\int_{0}^{\tau \wedge \widehat{\tau}} e^{-r u}\left[(\mu-r) \widehat{A}_{u}+r L\right] \mathrm{d} u\right] .
\end{aligned}
$$

Setting $\tau=T$ and observing

$$
V(A, T)=\mathbb{E}\left[e^{-r T} \widehat{A}_{T} \mathbf{1}_{\{T<\widehat{\tau}\}}\right],
$$

we can deduce the following relation between $F(A, T)$ and $V(A, T)$ :

$$
\begin{aligned}
V(A, T)= & A-F(A, T)-\mathbb{E}\left[L e^{-r \widehat{\tau}} \mathbf{1}_{\{T \geq \widehat{\tau}\}}\right] \\
& +(\mu-r) \mathbb{E}\left[\int_{0}^{T \wedge \widehat{\tau}} e^{-r u} \widehat{A}_{u} \mathrm{~d} u\right]
\end{aligned}
$$


We performed numerical calculations to explore the dependence of the expected present value of dividend payouts $F(A, T)$ on the ruin barrier $L$, dividend barrier $B$, volatility of the asset value process $\sigma$ and length of time horizon $T$. The plots in Figure 3 show that $F(A, T)$ is decreasing with respect to $L$ and $B$. Also, $F(A, T)$ is seen to be highly sensitive to the change in dividend barrier. From Figure 4, we observe that $F(A, T)$ is increasing with respect to $\sigma$ and $T$. All these results agree with our intuition on the behaviors of the expected present value of dividend payouts.

Another quantity of interest is the survival probability over a term $T$, as defined by

$$
S(A, T)=\mathbb{P}[\widehat{\tau}>T] .
$$

The partial differential equation formulation of $S(A, T)$ has been documented in Paulsen (2003). It is quite straightforward to establish the following relation between $V(A, T), F(A, T)$ and $S(A, T)$ :

$$
\begin{aligned}
V(A, T)= & A-F(A, T)+L e^{-r T} S(A, T)-L \mathbb{E}\left[e^{-r(T \wedge \widehat{\tau})}\right] \\
& +(\mu-r) \mathbb{E}\left[\int_{0}^{T \wedge \widehat{\tau}} e^{-r u} \widehat{A}_{u} \mathrm{~d} u\right] .
\end{aligned}
$$

In Figure 5, we show the plot of the survival probability $S(A, T)$ against $\ln (A / L)$ with varying values of the firm asset value volatility $\sigma$. A higher value of $\ln (A / L)$ indicates better creditworthiness of the firm, thus leading to a higher survival probability. On the other hand, a higher asset value volatility leads to a higher chance of hitting the ruin barrier and consequently a lower probability of survival.

\section{Conclusion}

In this paper, we derive the pricing formulation of the value function of the firm value process under the dividend barrier strategy and possibility of ruin. The upper dividend barrier is seen to be a reflecting barrier while the lower default barrier is an absorbing barrier. Our finite time dividend-ruin model resembles a path dependent option model with both the lookback and barrier features. We have presented the analytic formulations of the expected present value of the firm value, survival probability and expected present value of dividends over a finite time horizon. Closed form analytic solution to the value function of firm asset is obtained. In addition, a fairly 
accurate analytic approximation formula is also derived. The mathematical relations between the asset value function, expected present value of dividend payouts and survival probability of the finite time dividend-ruin model are presented. Our numerical calculations show that the asset value function, survival probability and dividend payouts are quite sensitive to the dividend barrier, firm's creditworthiness and volatility of firm value.

\section{Acknowledgement}

This research was supported by the Research Grants Council of Hong Kong, HKUST6425/05H.

\section{References}

Asmussen, S., Taksar, M., 1997. Controlled diffusion models for optimal dividend pay-out. Insurance: Mathematics and Economics 20, 1-15.

Borodin, A.N., Salminen, P., 2002. Handbook of Brownian motion - Facts and Formulae, second edition, Birkhäuser Verlag, Basel.

Choulli, T., Taksar, M., Zhou, X.Y., 2003. A diffusion model for optimal dividend distribution for a company with constraints on risk control. SIAM Journal of Control and Optimization 41, 1946-1979.

Chu, C.C., Kwok, Y.K., 2004. Reset and withdrawal rights in dynamic fund protection. Insurance: Mathematics and Economics 34, 273-295.

Crosbe, P.J., Bohn, J.R., 1993. Modeling default risk. Report issued by the KMV Corporation.

Domine, M., 1996. First passage time distribution of a Wiener process with drift concerning two elastic barriers. Journal of Applied Probability 33, 164-175.

Domine, M., 1996. First passage time distribution of a Wiener process with drift concerning two elastic barriers. Journal of Applied Probability 33, 164-175.

Freidlin, M., 1985. Functional Integration and Partial Differential Equations. Princeton University Press, Princeton, New Jersey, USA.

Gerber, H.U., Shiu, E.S.W., 2003. Geometric Brownian models for assets and liabilities: From pension funding to optimal dividends. North American Actuarial Journal 7(3), 37-56. 
Imai, J., Boyle, P., 2001. Dynamic fund protection. North American Actuarial Journal 5(3), 31-51.

Longstaff, F.A., Schwartz, E.S., 1995. A simple approach to valuing risky fixed and floating rate debts. Journal of Finance 50, 789-821.

Paulsen, J., Gjessing, H.K., 1997. Optimal choice of dividend barriers for a risk process with stochastic return on investments. Insurance: Mathematics and Economics 20, 215-223.

Paulsen, J., 2003. Optimal dividend payouts for diffusions with solvency constraints. Finance and Stochastics 7, 457-473.

Shreve, S.E., Lehoczky, J.P., Gaver, D.P, 1984. Optimal consumption for general diffusions with absorbing and reflecting barriers. SIAM Journal of Control and Optimization 22, 55-75.

Taksar, M., 2000. Optimal risk and dividend distribution control models for an insurance company. Mathematical Methods of Operations Research $51,1-42$. 
Appendix A - Proof of Eq. (3.2)

Under the assumption of $\bar{A}_{0}^{t}>B$, we obtain

$$
\begin{aligned}
A_{T} \min \left(1, \frac{B}{\bar{A}_{0}^{T}}\right) & =A_{T} \frac{B}{\max \left(\bar{A}_{0}^{t}, \bar{A}_{t}^{T}\right)} \\
& =A_{T} B \min \left(\frac{1}{\bar{A}_{0}^{t}}, \frac{1}{\bar{A}_{t}^{T}}\right) \\
& =A_{T} \frac{B}{\bar{A}_{0}^{t}} \min \left(1, \frac{B}{\bar{A}_{t}^{T}\left(\frac{B}{\bar{A}_{0}^{t}}\right)}\right) .
\end{aligned}
$$

Furthermore, assuming $\underline{\widehat{A}}_{0}^{t}>L$, we have

$$
\begin{aligned}
& 1_{\left\{\min _{0 \leq u \leq T}\left[A_{u} \min \left(1, \frac{B}{\bar{A}_{0}^{u}}\right)\right]>L\right\}} \\
= & 1_{\left\{\min _{t \leq u \leq T}\left[A_{u} \min \left(1, \frac{B}{\bar{A}_{0}^{u}}\right)\right]>L\right\}} \\
= & \mathbb{1}_{\left\{\min _{t \leq u \leq T}\left[\frac{A_{u} B}{\bar{A}_{0}^{t}} \min \left(1, \frac{B}{\bar{A}_{t}^{u} \frac{B}{\bar{A}_{0}^{t}}}\right)\right]>L\right\}} .
\end{aligned}
$$

Combining the results, when $\underline{\widehat{A}}_{0}^{t}>L$ and $\bar{A}_{0}^{t}>B$, we obtain

$$
\widetilde{A}_{T}=A_{T} \frac{B}{\bar{A}_{0}^{t}} \min \left(1, \frac{B}{\bar{A}_{t}^{T}\left(\frac{B}{\bar{A}_{0}^{t}}\right)}\right) \mathbf{1}_{\left\{\min _{t \leq u \leq T}\left[\frac{A_{u} B}{\bar{A}_{0}^{t}} \min \left(1, \frac{B}{\bar{A}_{t}^{u} \frac{B}{\bar{A}_{0}^{t}}}\right)\right]>L\right\}},
$$

and from which we can deduce the first relation in Eq. (3.2).

Appendix B - Green function with mixed Dirichlet-Neumann boundary conditions

Let $U(x ; y)$ denote the Laplace transform of $G(x, \tau ; y)$, where

$$
U(x ; y)=\int_{0}^{\infty} e^{-\gamma \tau} G(x, \tau ; y) \mathrm{d} \tau .
$$


The governing equation for $U(x ; y)$ is given by

$$
\frac{\sigma^{2}}{2} \frac{d^{2} U}{d x^{2}}+\alpha \frac{d U}{d x}-\gamma U=0, \quad \ell<x<0,
$$

with auxiliary conditions

$$
\begin{aligned}
U(\ell) & =U^{\prime}(0)=0, \\
U^{\prime}\left(y^{+}\right) & -U^{\prime}\left(y^{-}\right)=-\frac{2}{\sigma^{2}} \quad \text { and } \quad U\left(y^{+}\right)=U\left(y^{-}\right) .
\end{aligned}
$$

The solution to $U(x, y)$ is found to be

(i) $\ell<x<y$

$$
\begin{aligned}
U(x ; y) & D\left[(\beta-\alpha) e^{-\frac{\beta}{\sigma^{2}}(y-x+\ell)}+(\beta+\alpha) e^{-\frac{\beta}{\sigma^{2}}(\ell-x-y)}-(\beta-\alpha) e^{-\frac{\beta}{\sigma^{2}}(x+y-\ell)}-(\beta+\alpha) e^{-\frac{\beta}{\sigma^{2}}(x-y-\ell)}\right]
\end{aligned}
$$

(ii) $y<x<0$

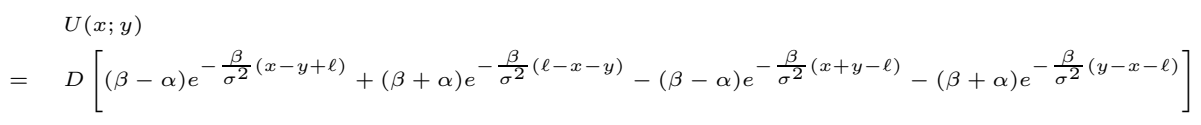

where $D=\frac{e^{\frac{\alpha}{\sigma^{2}}(y-x)}}{2 \beta\left[\beta \cosh \left(\frac{\beta \ell}{\sigma^{2}}\right)+\alpha \sinh \left(\frac{\beta \ell}{\sigma^{2}}\right)\right]}$ and $\beta=\sqrt{\alpha^{2}+2 \gamma \sigma^{2}}$. The following Laplace inversion formula [see p.642, Borodin and Salminen (2002)] is useful:

$$
\begin{aligned}
& \mathcal{L}_{\gamma}^{-1}\left(\frac{(2 \gamma)^{\widehat{\mu} / 2} e^{-\widehat{x} \sqrt{2 \gamma}}}{\sinh (\widehat{t} \sqrt{2 \gamma})+z \sqrt{2 \gamma} \cosh (\widehat{t} \sqrt{2 \gamma})}\right) \\
= & \sum_{k=0}^{\infty} \frac{(-1)^{k} k !}{2^{k} z^{k+1}} \sum_{\ell=0}^{k} \frac{(-1)^{\ell}}{(k-\ell) ! \ell !} c_{\widehat{y}}(\widehat{\mu}-k-1, k+1, \widehat{t}, \widehat{x}+k \widehat{t}-2 \widehat{\ell t}), \quad \widehat{x}>-\widehat{t},
\end{aligned}
$$

where $\gamma$ is the dummy Laplace variable, $z \neq 0, \widehat{t}>0$ and the function $c_{\widetilde{y}}$ is defined by

$$
\begin{aligned}
& c_{\widetilde{y}}(\widetilde{\mu}, \widetilde{\nu}, \widetilde{t}, \widetilde{z}) \\
&= 2^{\widetilde{\nu}} \sum_{j=0}^{\infty} \frac{(-1)^{j} \Gamma(\widetilde{\nu}+j) e^{-(\widetilde{\nu} \widetilde{t}+2 j \widetilde{t})^{2} /(4 \widetilde{y})}}{\sqrt{2 \pi} \widetilde{y}^{1+\frac{\tilde{\mu}}{2}} \Gamma(\widetilde{\nu}) j !} D_{\widetilde{\mu}+1}\left(\frac{\widetilde{\nu} \widetilde{t}+\widetilde{z}+2 j \widetilde{t}}{\sqrt{\widetilde{y}}}\right), \\
& \widetilde{\nu} \geq 0, \widetilde{\nu}+\widetilde{t}>0 \quad \text { and } \quad \widetilde{t}>0,
\end{aligned}
$$


$D_{\widetilde{\mu}+1}$ is the parabolic cylinder function. One can then use the inversion formula to perform the Laplace inversion of $U(x ; y)$ to obtain $G(x, \tau ; y)$.

\section{Appendix C - Derivation of analytic approximation formula (3.11)} For the Brownian motion $W_{t}$ with drift $\alpha$ and variance rate $\sigma^{2}$, we write $\underline{W}_{0}^{T}=\min _{0 \leq t \leq T} W_{t}$ and $\bar{W}_{0}^{T}=\max _{0 \leq t \leq T} W_{t}$, the density function $\widehat{f}\left(x, M, T ; x_{0}\right)$ of the terminal value $W_{T}$ conditional on $W_{0}=x_{0}$ and subject to lower and upper absorbing barriers at $x=0$ and $x=M$, respectively, has been presented in Eq. (3.13). The joint density of $W_{T}$ and $\bar{W}_{0}^{T}$ is given by

$$
\begin{aligned}
& \widehat{f}\left(x, M, T ; x_{0}\right) \mathrm{d} x \mathrm{~d} M \\
= & \mathbb{P}\left[W_{T} \in \mathrm{d} x, \bar{W}_{0}^{T} \in \mathrm{d} M, \underline{W}_{0}^{T}>0 \mid W_{0}=x_{0}\right] \\
= & \frac{\partial f}{\partial M}\left(x, M, T ; x_{0}\right) \mathrm{d} x \mathrm{~d} M .
\end{aligned}
$$

We write $A=A(0), K=\ln \frac{B}{L}$ and let $x_{0}=W_{0}=\ln \frac{A}{L}$ so that $x_{0}=0$ when $A=L$. The restricted asset value process is given by

$$
\begin{aligned}
V_{a}(A, T ; L, B)= & \mathbb{E}\left[e^{-r T} L e^{W_{T}} \min \left(1, \frac{B}{L e^{\bar{W}_{0}^{T}}}\right) \mathbf{1}_{\left\{\underline{W}_{0}^{T}>0\right\}}\right] \\
= & e^{-r T}\left[L \int_{0}^{K} \int_{0}^{M} e^{x} \widehat{f}\left(x, M, T ; x_{0}\right) \mathrm{d} x \mathrm{~d} M\right. \\
& \left.+B \int_{K}^{\infty} \int_{0}^{M} e^{x} e^{-M} \widehat{f}\left(x, M, T ; x_{0}\right) \mathrm{d} x \mathrm{~d} M\right] .
\end{aligned}
$$

We let

$$
\begin{aligned}
I_{1} & =\int_{0}^{K} \int_{0}^{M} e^{x} \widehat{f}\left(x, M, T ; x_{0}\right) \mathrm{d} x \mathrm{~d} M \\
& =\int_{0}^{K} \int_{x}^{K} e^{x} \frac{\partial f}{\partial M} \mathrm{~d} M \mathrm{~d} x \\
& =\int_{0}^{K} e^{x}\left[f\left(x, K, T ; x_{0}\right)-f\left(x, x, T ; x_{0}\right)\right] \mathrm{d} x
\end{aligned}
$$


and

$$
\begin{aligned}
I_{2}= & \int_{K}^{\infty} \int_{0}^{M} e^{x} e^{-M} \frac{\partial f}{\partial M} \mathrm{~d} x \mathrm{~d} M \\
= & \int_{0}^{K} \int_{K}^{\infty} e^{x}\left[\frac{\partial}{\partial M}\left(e^{-M} f\right)+e^{-M} f\right] \mathrm{d} M \mathrm{~d} x \\
& +\int_{K}^{\infty} \int_{x}^{\infty} e^{x}\left[\frac{\partial}{\partial M}\left(e^{-M} f\right)+e^{-M} f\right] \mathrm{d} M \mathrm{~d} x \\
= & -\int_{0}^{K} e^{x} \frac{L}{B} f\left(x, K, T ; x_{0}\right) \mathrm{d} x-\int_{K}^{\infty} f\left(x, x, T ; x_{0}\right) \mathrm{d} x \\
& +\int_{K}^{\infty} \int_{0}^{M} e^{x} e^{-M} f\left(x, M, T ; x_{0}\right) \mathrm{d} x \mathrm{~d} M .
\end{aligned}
$$

It is easily seen that $f\left(x, x, T ; x_{0}\right)=0$. Combining the above results together, we obtain

$$
V_{a}(A, T ; L, B)=e^{-r T} B \int_{K}^{\infty} \int_{0}^{M} e^{x} e^{-M} f\left(x, M, T ; x_{0}\right) \mathrm{d} x \mathrm{~d} M .
$$




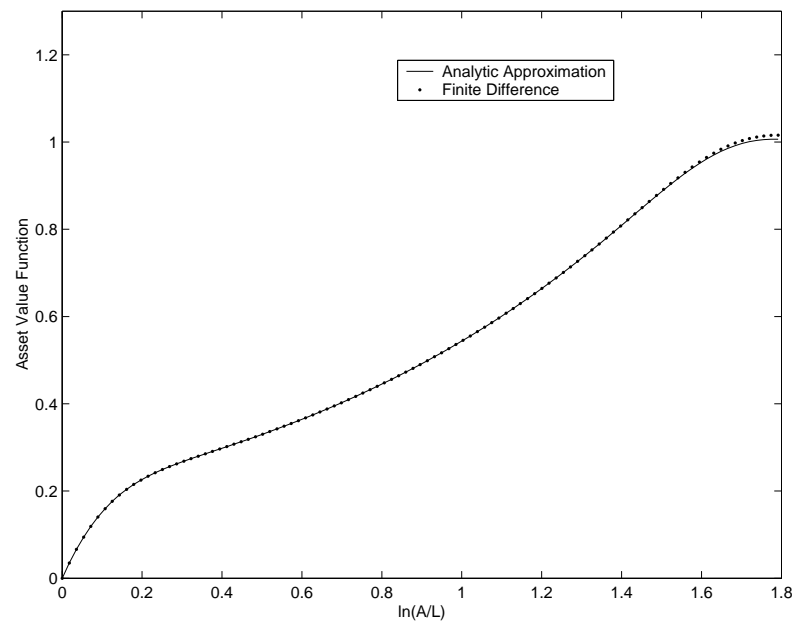

Fig. 1. Plot of the asset value function $V(A, T ; L, B)$ against $\ln (A / L)$. The asset value is an increasing function of the firm's creditworthiness [as measured by $\ln (A / L)]$. The finite difference solution agrees very well with the solution obtained by the analytic approximation formula.

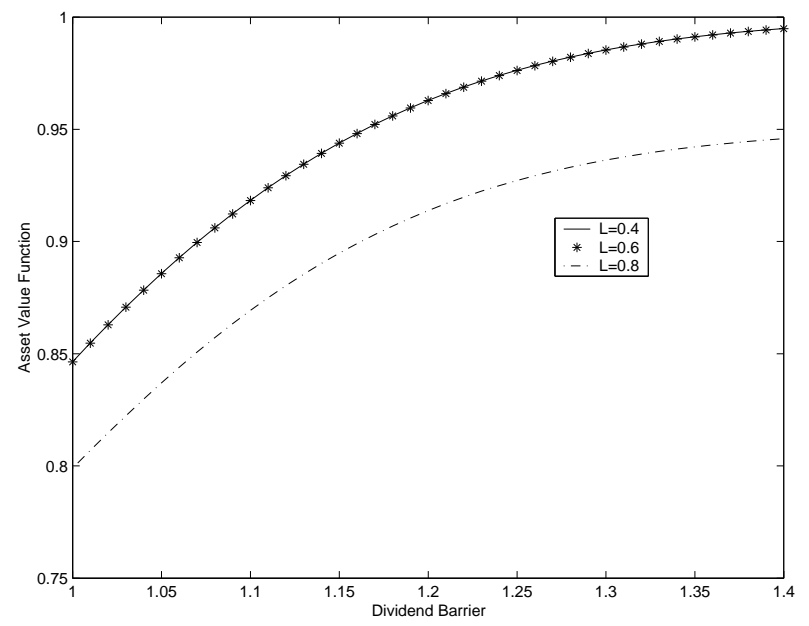

Fig. 2. Plot of the asset value function $V(A, T ; L, B)$ against the dividend barrier $B$ with varying values of the ruin barrier $L$. When the credit quality of the firm is relatively high, the asset value function is not quite sensitive to the level of ruin barrier. However, for a fixed value of $L$, the asset value function shows a relatively strong dependence on the dividend barrier. 


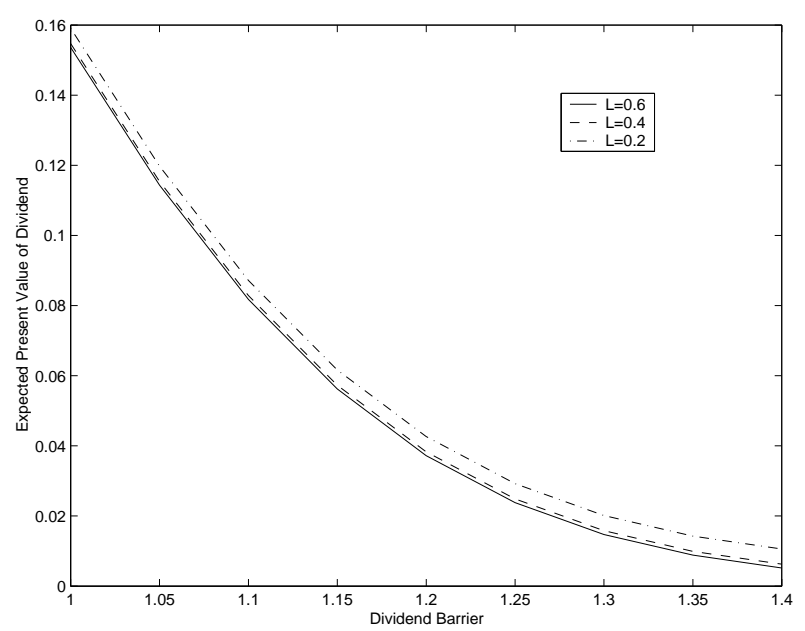

Fig. 3. Plot of the expected present value of dividend payouts $F(A, T)$ against the dividend barrier $B$ with varying values of ruin barrier $L$. The expected present value of dividend is a decreasing function of $L$ and $B$.

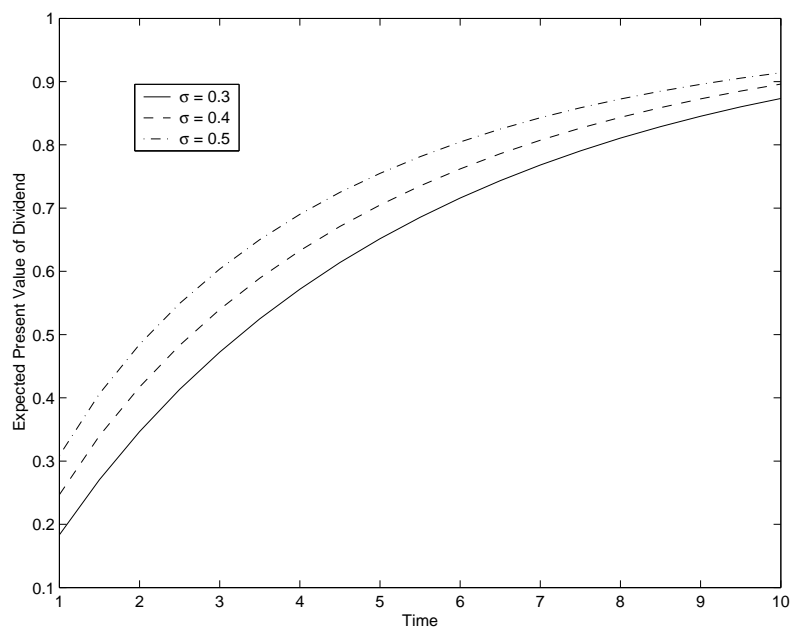

Fig. 4. Plot of the expected present value of dividend payouts $F(A, T)$ against the length of time horizon $T$ with varying values of firm asset value volatility $\sigma$. The expected present value of dividend is an increasing function of the length of time horizon and volatility of asset value. 


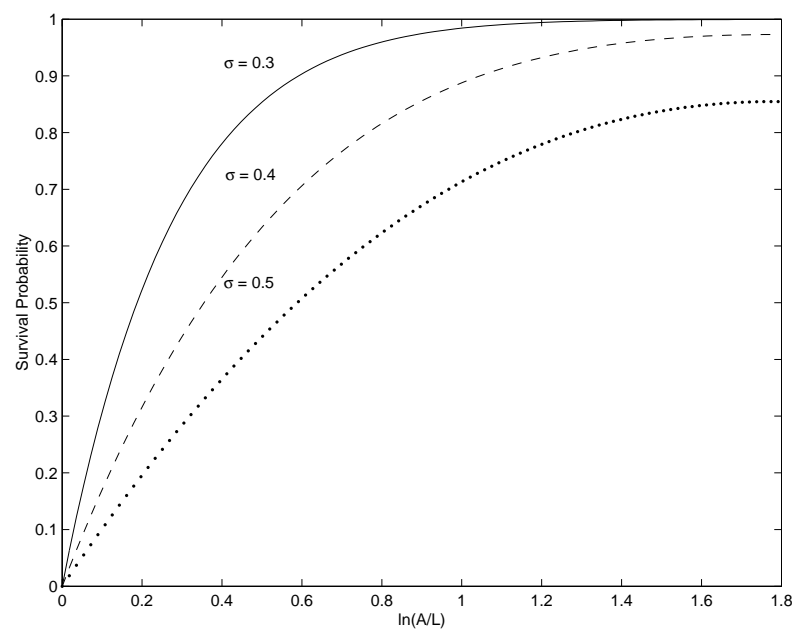

Fig. 5. Plot of the survival probability $S(A, T)$ against $\ln (A / L)$ with varying values of the firm asset value volatility $\sigma$. The survival probability is an increasing function of the creditworthiness of firm and a decreasing function of volatility. 